Deutschen Industrie erhalten. Der BDI befürchtete unter anderem eine gesamtgesellschaftliche Mitbestimmung der technischen Entwicklung, die von einer nach politischen Gesichtspunkten besetzten Einrichtung für Technikfolgenabschätzung ausginge. Auch werde die parlamentarische Arbeit eher entlastet als erleichtert. Der Industrieverband hielt eine reine Clearingstelle für die bessere Lösung. Ihre Hauptaufgabe sollte es sein. die Informationsflut im technisch-wissenschaftlichen Bereich $\mathrm{zu}$ bündeln und zu problemgerechten Fragen der Parlamentarier aufzubereiten. Dies entspräche aber in keiner Weise dem, was sich Abgeordnete aller Fraktionen vorstellten und worauf sie jahrelang hingearbeitet haben. Sie wollen eine engere Verknüpfung des wissenschaftlichen Sachverstandes mit der Politik.

\section{Neue Zentren für Molekularbiologie}

Das System der Konzentration der Genforschung in gut ausgestatteten Zentren (Naturwissenschaften 11/1986) wurde Ende des vergangenen Jahres um drei weitere Aktivitäten ergänzt. Zu den drei Genzentren in Heidelberg, Köln und München ist ein viertes getreten, das Institut für Genbiologische Forschung Berlin GmbH (IGF). An der Universität Hamburg wird ein Zentrum für Molekularbiologie aufgebaut, und in Darmstadt wurde eine Arbeitsgemeinschaft Gentechnik gegründet.

Gesellschafter in der neuartigen Konstruktion des IGF sind das Land Berlin und die Schering AG. Mit der Freien Universität Berlin wurde Zusammenarbeit vereinbart, der wissenschaftliche Geschäftsführer des IGF, Prof. L. Willmitzer, ist zugleich Lehrstuhlinhaber für Molekularbiologie an der FU, wo auch ein eigener Studiengang Biochemie eingerichtet wurde. Die neue Einrichtung konzentriert ihre wissenschaftlichen Arbeiten auf zwei Schwerpunkte: molekularbiologische und zellbiologische Untersuchungen an höheren Pflanzen, speziell die Kontrolle von deren Genaktivitäten - insbesondere am Beispiel der Kartoffel -, und molekulargenetische Untersuchungen an Mikroorganismen, zum Beispiel die Wechselwirkungen zwischen höheren Pflanzen und Pilzen. Neben den beiden ständigen Abteilungen sollen vier bis sechs wissenschaftlich unabhängige Arbeitsgruppen von jüngeren Wissenschaftlern gebildet werden. Die Schering AG begründet ihr Interesse an dem Institut mit seiner Aufgabe als Forum des wissenschaftlichen Informationsaustausches, als Quelle für qualifizierten wissenschaftlichen Nachwuchs und als $\mathrm{Zu}$ gangsmöglichkeit zu neuen Methoden und Erkenntnissen. Das Unternehmen hat eine Option auf vom Institut erworbene Schutzrechte zu marktüblichen Bedingungen.

Befürchtungen, das neue Institut könne in seiner wissenschaftlichen Arbeit von außen beeinflußt werden, tritt Willmitzer mit dem Hinweis auf die Funktion der ver- schiedenen Entscheidungsebenen entgegen. Die Gesellschaftsversammlung hat neben den gesetzlichen vorgeschriebenen Verwaltungsaufgaben Einflu $ß$ auf Finanzrahmen und Finanzverwaltung. Das von ihr gewählte Kuratorium besteht aus jeweils vom Land Berlin und der Schering AG vorgeschlagenen Mitgliedern sowie fünf unabhängigen Wissenschaftlern. Es hat wissenschaftlich beratende Funktion und ist gleichzeitig weisungsbefugter Aufsichtsrat. Die Geschäftsführung hat Führungs- und Entscheidungsaufgaben ,gemä $\beta$ dem Gesellschaftsvertrag sowie den Beschlüssen des Kuratoriums", zusätzliche Vereinbarungen, welche die wissenschaftliche Unabhängigkeit einschränken, gebe es nicht, meinte Willmitzer in einem Interview mit dem Informationsblatt der FU; ,die Ausrichtung der wissenschaftlichen Arbeiten wird vom Institut bestimmt".

Das Zentrum für Molekularbiologic der Universität Hamburg wird mit massiver finanzieller Hilfe des Bundesforschungsministeriums aufgebaut und besteht aus zwei Schwerpunkten. Die Molekulare Neurobiologie am Universitätskrankenhaus. Eppendorf umfaßt sechs Abteilungen: Zellbiochemie und klinische Neurobiologie (schon seit Anfang 1985), Struktur und Funktion neuronaler Membranproteine, Neuronale Entwicklung und Regeneration sowie Signalverarbeitung, Molekulare Neuroanatomie und -pathologie. Das Land Hamburg schafft für diesen Schwerpunkt 50 neue Stellen. Das BMFT finanziert zusätzlich zehn bis zwölf Jahre lang 40 bis 50 auf fünf Jahre befristete Stellen für fünf Nachwuchsforschergruppen nach dem Vorbild des Genzentrums München und beteiligt sich an den Anfangsinvestitionen und den allgemeinen Kosten. Es will diesen Schwerpunkt insgesamt mit bis zu 5 Mio DM jährlich fördern. Während sich der Schwerpunkt Neurobiologie zur Beschleunigung des Anfangs zunächst in Forschungscontainern untergebracht - mit Fragen der Gehirn- und Nervenfunktion befaßt, ist es Ziel des Schwerpunkts ,Biotechnologie/Angewandte Molekularbiologie der Pflanzen", aus Erkenntnissen der Grundlagenforschung neue biotechnische Methoden im Bereich der Pflanzenzüchtung zu entwickeln. Er besteht aus den Forschungsrichtungen Molekulare Genetik/Gentechnologie, Zellkultur und Molekulare Phytopathologie.

Für diesen Schwerpunkt, der im Institut für Allgemeine Botanik untergebracht ist, sind 16,5 Stellen vorgesehen. Das BMFT finanziert drei Arbeitsgruppen: einschließlich der Anfangsinvestitionen betragen die jährlichen Fördermittel aus Bonn bis zu 3 Mio DM.

An der Arbeitsgemeinschaft Gentechnik in Darmstadt sind das Fachgebiet Biochemie der Technischen Hochschule sowie vier pharmazeutische Firmen (Grünenthal GmbH, Aachen, E. Merck, Röhm GmbH und Röhm Pharma, alle Darmstadt) beteiligt. Die Projekte der Arbeitsgemeinschaft, die nach dem Modell der Verbundforschung von den Firmen und dem BMFT gefördert werden, sollen rasche Umsetzung der Ergebnisse der Grundlagenforschung ermöglichen.

Verantwortlich für den Anzeigenteil: E. Lückermann, Heidelberger Platz 3, D-1000 Berlin 33. - C Springer-Verlag, Berlin-Heidelberg 1987.

Springer-Verlag GmbH \& Co. KG, D-1000 Berlin 33. - Gesamtherstellung: Universitätsdruckerei H. Stürtz AG, Würzburg. - Printed in Germany. 